\title{
Crecimiento postraumático en hombres gays con VIH en Buenos Aires, Argentina
}

\author{
Post-traumatic growth among gay men living with HIV \\ from Buenos Aires, Argentina
}

\author{
Pablo David Radusky ${ }^{1,2}$, Virginia Zalazar ${ }^{2}$ e Inés Aristegui ${ }^{2,3}$ \\ Universidad de Buenos Aires ${ }^{1}$ \\ Fundación Huésped ${ }^{2}$ \\ Universidad de Palermo ${ }^{3}$
}

Autor para correspondencia: Pablo David Radusky,pradusky@psi.uba.ar.

\section{RESUMEN}

\begin{abstract}
Este estudio cualitativo tuvo como objetivo explorar los significados atribuidos al virus de inmunodeficiencia humana (VIH) y la experiencia de cambios positivos posteriores al diagnóstico (crecimiento postraumático) en hombres gays de Buenos Aires, Argentina. Se efectuaron catorce entrevistas individuales en profundidad. Se implementó un análisis temático asignando códigos a fragmentos de transcripciones y organizándolos en temas. La edad media de los participantes fue de 41.29 (D.E. $=11.97$ ) y una media de 8.42 (D.E. $=7.08$ ) años desde el diagnóstico, tres de los cuales lo habían recibido en el último año. La mayoría informó cambios positivos que atribuyeron al diagnóstico de VIH, los que se organizaron en tres temas: Cambios en la filosofía de vida, Cambios en las relaciones con los otros y Cambios en la percepción de sí mismos. Algunos de estos incluyen mayor aprecio por la vida y su salud, incremento de comportamientos más saludables, fortalecimiento de la red de apoyo social y conciencia de sus capacidades para el afrontamiento. Tales cambios parecen asociarse a la atribución al VIH de sentidos más favorables. La identificación de crecimiento postraumático puede orientar intervenciones psicoterapéuticas y psicosociales dirigidas a hombres gays con VIH en virtud de que funciona como un recurso para afrontar el estigma y se asocia con una mayor retención en la atención del VIH.
\end{abstract}

Palabras clave: Crecimiento postraumático; Hombres gays; VIH; Argentina; Investigación cualitativa.

\begin{abstract}
This qualitative study aimed to explore the meanings attributed to HIV and the experimentation of positive changes after diagnosis (post-traumatic growth) in gay men from Buenos Aires, Argentina. Fourteen individual, in-depth interviews were thematically analyzed by assigning codes to fragments of transcripts and organizing them along with themes. The mean age of participants was 41.29 years, with a mean of 8.42 years since diagnosis, three of them diagnosed in the last year. Most participants reported positive changes attributed to their HIV diagnosis, organized into three themes: a) changes in their philosophy of life, b) changes in their relations with others, and c) changes in self-perception. Some of these include greater appreciation for life and their own health, increments in healthier behaviors, strengthening of the social support network, and awareness of
\end{abstract}

\footnotetext{
${ }^{1}$ Facultad de Psicología, Av. Independencia 3065 (C1225AAM), tel. (54)11- 52-87-32-00, Buenos Aires, Argentina, correos: pablo.radusky@ huesped.org.ar/pradusky@psi.uba.ar.

${ }^{2}$ Dirección de Investigaciones, Dr. Carlos Gianantonio (ex Pje. Peluffo) 3932, (C1202ABB), Buenos Aires, Argentina, tel. (54)11-49-81-77-77, correo: virginia.zalazar@huesped.org.ar.

${ }^{3}$ Mario Bravo 1050 (C1175ABT), Buenos Aires, Argentina, tel. (54)11-49-64-46-00, correo: ines.aristegui@huesped.org.ar.
} 
their coping skills. Such changes seem to be associated with the attribution of more positive meanings to HIV. The identification of post-traumatic growth can guide psychotherapeutic and psychosocial interventions in gay men living with HIV because it functions as a resource to cope with stigma and is associated with greater adherence to HIV care.

Key words: Post-traumatic growth; Gay men; HIV; Argentina; Qualitative research.

Recibido: 09/11/2020

Aceptado: 18/01/2021

$\mathrm{S}$ e estima que, en Argentina, 139 mil personas viven con el virus de la inmunodeficiencia humana (VIH). Sin embargo, la prevalencia es especialmente elevada en algunos grupos específicos, como los hombres gays y otros hombres que tienen sexo con hombres (HSH). En este grupo, las cifras se estiman entre 12 y $15 \%$, en comparación con una prevalencia en la población general de $0.4 \%$. En los últimos años se ha registrado un aumento de la prevalencia entre adolescentes y jóvenes gays de 15 a 24 años (Ministerio de Salud de la Nación, 2019).

Recibir un diagnóstico de VIH conlleva un cambio en el status psicosocial, pues ubica a los sujetos frente a nuevos desafíos, como el inicio de un tratamiento crónico y la posibilidad de experimentar discriminación y estigmatización (Yu, Chen, Ye, Li y Lin, 2017). Por este motivo, puede ser vivido como un evento altamente estresante que tiene un impacto emocional significativo e incluso potencialmente traumático (Martin y Kagee, 2011; Nightingale, Sher y Hansen, 2010; Theuninck, Lake y Gibson, 2010). Por consiguiente, el diagnóstico de VIH se halla estrechamente asociado a un elevado malestar emocional con consecuencias adversas sobre la salud mental (Adams, Zacharia, Masters, Coffey y Catalan, 2016). Ello se explica en parte por el hecho de que los significados específicos asociados con el VIH y la valoración que el sujeto hace del mismo pueden amenazar o discrepar con sus creencias y presunciones globales acerca de, por ejemplo, la justicia y el

Instituto de Investigaciones Psicológicas - Universidad Veracruzana ISSN impreso: 1405-1109 mérito, sus metas en la vida y el sentido que le atribuye a su propia existencia (Park, 2013; Tedeschi y Calhoun, 2004).

Es por este motivo que la investigación acerca del impacto psicológico del VIH ha estado más focalizada en las consecuencias negativas que entraña para la salud mental. No obstante, algunos autores han señalado que la trayectoria resiliente frente a las situaciones traumáticas es, de hecho, mucho más frecuente (Bonanno, 2004). Por tal razón, la psicología ha comenzado a reorientar su foco de atención para explorar los recursos que los individuos poseen para enfrentar constructivamente la adversidad y superarla de forma adaptativa y saludable. En esta línea, Tedeschi y Calhoun (2004) han postulado que el malestar producido al enfrentarse a situaciones traumáticas o altamente estresantes puede impulsar ciertos cambios psicológicos positivos, conocidos como crecimiento postraumático, o CPT. Dicho crecimiento implica el desarrollo o la mejora de algunas áreas de la vida de los sujetos que supera incluso los niveles existentes antes de la crisis o el evento traumático. Según estos autores, tales cambios pueden agruparse en cinco dominios: mayor aprecio por la vida y reorganización de prioridades, cambios en la relación con los otros, mayor sentido de fortaleza personal, cambios espirituales y descubrimiento de nuevas posibilidades en la vida.

Un creciente cúmulo de evidencias muestra que la mayoría de las personas con VIH, incluidos los hombres gays, experimentan algún grado de CPT como consecuencia del diagnóstico (Garrido, Murphy y Alonso, 2017; Milam, 2006; Murphy y Hevey, 2013; Siegel y Schrimshaw, 2000a). En un estudio cualitativo, Siegel y Schrimshaw (2000b) hallaron seis conjuntos de cambios positivos experimentados por las personas con VIH que pueden encuadrarse dentro del CPT: incrementos en la espiritualidad y la religiosidad, mejoras en las relaciones interpersonales, cambios positivos en la visión de sí mismos, mayor aprecio y valoración por la vida, reorganización de metas y prioridades e incremento de las conductas saludables. La evidencia muestra que el CPT está asociado a una mayor salud mental y a mejoras en la salud física de las personas con VIH (Milam, 2004, 2006; Rzeszutek, Oniszczenko y Firląg-Burkacka, 2016; Sawyer, 
Ayers y Field, 2010; Willie et al., 2016). En hombres gays y otros $\mathrm{HSH}$, los cambios incluidos dentro del CPT funcionan como estrategias de afrontamiento ante el VIH, moderando el estrés producido por el diagnóstico y promoviendo un mayor ajuste psicológico (Arístegui, Radusky, Zalazar, Lucas y Sued, 2017; Siegel y Schrimshaw, 2000a).

En este sentido, el CPT y el malestar producido por el evento adverso, como un diagnóstico de VIH, pueden coexistir, por lo que los individuos pueden experimentar cambios positivos, aunque continúen experimentando niveles significativos de emocionalidad negativa en forma simultánea (Tedeschi y Calhoun, 2004; Yu et al., 2017). El CPT es activado precisamente por el intento de reducir el malestar que genera la discrepancia entre los sentidos atribuidos al diagnóstico y las creencias globales del sujeto (Park, 2013). Con el fin de reducir dicha discrepancia, el sujeto puede modificar los sentidos específicos atribuidos al VIH y revalorizarlo de forma más favorable para que concuerde y pueda ser asimilado al sistema general de creencias anterior al diagnóstico (Fife, 2005). Otorgar sentidos positivos a una situación adversa se asoció a CPT en hombres gays jóvenes con diagnóstico reciente de VIH (Yu et al., 2017). Es por este motivo que los significados que las personas atribuyen al VIH cobran particular relevancia en relación con el desarrollo del CPT y el ajuste psicológico y adaptación posteriores al diagnóstico (Fife, 2005; Sawyer et al., 2010).

De lo anterior se desprende que el CPT es un fenómeno especialmente relevante respecto a la salud mental de las personas con VIH, y en particular de los hombres gays. Desde un enfoque interseccional, los hombres gays con VIH pueden experimentar múltiples formas de estigma y discriminación (relacionadas con el VIH y con su orientación sexual, por ejemplo) que interactúan y se potencian entre sí, intensificando sus consecuencias adversas sobre la salud mental (Jeffries et al., 2015; Radusky y Mikulic, 2019). En Argentina, la aprobación de la Ley $\mathrm{N}^{\circ} 26.618$ de Matrimonio Igualitario (2010) (cf. Krasnow, 2012) ha promovido un clima social de mayor aceptación hacia las personas gays y de orientación sexual diversa. Sin embargo, algunas formas de estigma y discriminación por orientación sexual aún persisten (Federación Argentina LGBT, 2019). Frente a esto, los cambios comprendidos por el CPT resultan un factor protector (Arístegui et al., 2017). De modo similar, una mayor salud mental y bienestar psicológico se asocian a una mayor adherencia al tratamiento antirretroviral y a la retención en la atención del VIH (Bassett, Schuette, O’Dwyer y Moskowitz, 2019; Camargo et al., 2019). La importancia de lo anterior radica en que una mayor adherencia al tratamiento implica asimismo una mayor probabilidad de alcanzar una carga viral indetectable, lo que favorece la salud y la calidad de vida de las personas con VIH y evita el pasaje a la fase de sida.

No obstante, la evidencia acerca de la ocurrencia de CPT en hombres gays con VIH es escasa en Latinoamérica. Por consiguiente, el presente estudio se propuso explorar los sentidos atribuidos al VIH y la experimentación de cambios positivos posteriores al diagnóstico que pueden encuadrarse dentro del concepto de CPT en hombres gays residentes en el área metropolitana de la ciudad de Buenos Aires, Argentina.

\section{MÉTODO}

\section{Participantes}

La muestra estuvo conformada por 14 hombres que se identificaban como gays cisgénero $^{4}$, quienes reportaron vivir con VIH y residir en Buenos Aires (Tabla 1), y cuya media de edad fue de 41.29 años (D.E. =11.97). Todos los participantes informaron haber adquirido el VIH por vía sexual. La media de años transcurridos desde el diagnóstico fue de 8.42 años (D.E. = 7.08), al momento de la entrevista, y tres participantes lo habían recibido dentro de los doce meses anteriores a la misma, por lo que podrían considerarse recientes, según el criterio de Wang et al. (2017). Excepto uno, todos ellos se encontraban recibiendo tratamiento antirretroviral.

\footnotetext{
${ }^{4}$ Cisgénero es una identidad de género opuesta a transgénero. Una persona es cisgénero si se identifica con el género asignado al nacer.
} 
Tabla 1. Características de los participantes.

\begin{tabular}{|c|c|c|c|}
\hline Participante & Edad & $\begin{array}{c}\text { Nivel } \\
\text { educativo }\end{array}$ & $\begin{array}{c}\text { Años desde } \\
\text { el diagnóstico }\end{array}$ \\
\hline 1 & 45 & 3 & 16 \\
\hline 2 & 40 & 1 & 15 \\
\hline 3 & 44 & 3 & $-{ }^{\mathrm{b}}$ \\
\hline 4 & 41 & 3 & $--^{\mathrm{b}}$ \\
\hline 5 & 42 & 3 & 20 \\
\hline 6 & 25 & 2 & 7 \\
\hline 7 & 49 & 3 & 16 \\
\hline 8 & 27 & 1 & 8 \\
\hline 9 & 40 & 1 & 4 \\
\hline 10 & 49 & 3 & 4 \\
\hline 11 & 71 & 3 & 4 \\
\hline 12 & 25 & 2 & 5 \\
\hline 13 & 33 & 3 & 3 \\
\hline 14 & 47 & 3 & \\
\hline
\end{tabular}

Nota: $\mathrm{a}=1$ : Secundario completo, 2: Terciario o Universitario incompleto, 3: Terciario o Universitario completo; $\mathrm{b}=$ Tiempo desde el diagnóstico inferior a un año.

\section{Instrumentos}

Dado que se optó por el uso de una metodología cualitativa, la recolección de información se efectuó mediante entrevistas en profundidad individuales semiestructuradas. Para la aplicación de esta técnica se elaboró una guía de entrevista en la que se predefinieron las áreas a explorar, que ya fueron descritas y detalladas en otro lugar (Radusky y Mikulic, 2018, 2019). En el presente trabajo se expone el análisis de las respuestas a una de tales áreas de exploración, que implicó la indagación sobre los cambios positivos o negativos que los participantes reconocían haber experimentado como consecuencia del diagnóstico de VIH.

\section{Procedimiento}

Esta investigación se llevó a cabo en concordancia con los estándares éticos de la institución en que se realizó. Antes de comenzar cada entrevista, todos los participantes fueron informados de los objetivos y procedimientos del estudio, así como de la confidencialidad de la información que proporcionaran. A continuación se les solicitó su consentimiento para participar voluntariamente en esta investigación y para que las entrevistas fueran audiograbadas.

En primera instancia, se estableció contacto con tres participantes que coordinaban grupos de pares en una organización no gubernamental de la ciudad, a los que se entrevistó y fueron los encargados de difundir la invitación a participar en el estudio entre los asistentes a los citados grupos, poniendo a los interesados en contacto con los investigadores. Una vez que estos nuevos participantes fueron entrevistados, se les propuso que informasen sobre el estudio a otras personas que cumplieran los criterios de inclusión para invitarlos a participar. Este procedimiento se repitió hasta que se alcanzó la saturación de la información recabada, por lo que se detuvo el proceso.

A continuación, se reemplazó la identidad de cada participante por un número asignado a cada entrevista para resguardar la confidencialidad de la información proporcionada, omitiendo todos los datos de identidad o que permitiesen relacionar fragmentos del discurso con personas particulares. Todas las entrevistas fueron hechas entre octubre de 2015 y agosto de 2016, y luego grabadas y transcritas por el mismo investigador, siendo su duración en general de media hora a una hora y media, aproximadamente.

\section{Análisis de datos}

Se realizó un análisis temático del material transcrito (Braun y Clarke, 2006), asistido por el software para análisis cualitativo ATLAS.Ti, versión 7.5.4 (2015). Primeramente, se llevó a cabo un proceso de codificación abierta, mediante el cual se asignó a cada fragmento significativo de material un código que lo representara. Luego, se identificaron y establecieron relaciones entre códigos conceptualmente asociados, agrupándolos en temas o ejes teóricos de orden superior. Se tuvieron en cuenta los temas que se reiteraron a lo largo de la mayoría de las entrevistas. Finalmente, se efectuó un proceso de recodificación yendo de los ejes a los códigos. Como resultado, la definición de los códigos y su asignación a fragmentos de material fueron refinadas para hacerlas más precisas. Asimismo, se desarrollaron nuevos códigos 
cuando se consideró necesario (por ejemplo, se fusionaron códigos conceptualmente equivalentes para simplificar la codificación, y los códigos demasiado amplios y heterogéneos se desagregaron en otros más precisos y concretos). Esto consolidó los ejes temáticos propuestos, los que pueden apreciarse en la Tabla 2 junto con sus principales códigos.

Tabla 2. Temas o ejes y principales códigos.

\begin{tabular}{|c|c|c|}
\hline Temas & Subtemas & Ejemplos de códigos \\
\hline $\begin{array}{l}\text { 1. Sentidos atribuidos } \\
\text { al diagnóstico de VIH. }\end{array}$ & & $\begin{array}{l}\text { 1.1. Segunda oportunidad } \\
\text { 1.2. Aprendizaje } \\
\text { 1.3. Situación límite } \\
\text { 1.4. Culpa y responsabilidad } \\
\text { 1.5. Desdramatización/naturalización } \\
\text { 1.6. Normalización }\end{array}$ \\
\hline \multirow{3}{*}{$\begin{array}{l}\text { 2. Cambios positivos atribuidos } \\
\text { al diagnóstico de VIH. }\end{array}$} & $\begin{array}{l}2.1 \text { Cambios en la filosofía } \\
\text { de vida. }\end{array}$ & $\begin{array}{l}\text { 2.1.1. Valor de la vida y el tiempo } \\
\text { 2.1.2. Cambios en las actividades y en las prioridades } \\
\text { 2.1.3. Valor del cuerpo y la salud } \\
\text { 2.1.4. Incremento en el cuidado de la propia salud }\end{array}$ \\
\hline & $\begin{array}{l}\text { 2.2 Cambios vinculares } \\
\text { y en la relación con los otros. }\end{array}$ & $\begin{array}{l}\text { 2.2.1. Cambios en la priorización de los vínculos y mayor } \\
\text { expresión e intercambio emocional } \\
\text { 2.2.2. Fortalecimiento de vínculos cercanos y significativos } \\
\text { 2.2.3. Fortalecimiento de la red de apoyo: aceptación de la } \\
\text { ayuda de otros }\end{array}$ \\
\hline & $\begin{array}{l}2.3 \text { Cambios en la percep- } \\
\text { ción de sí mismos. }\end{array}$ & $\begin{array}{l}\text { 2.3.1. Nueva conciencia de las propias fortalezas y recursos } \\
\text { para el afrontamiento } \\
\text { 2.3.2. Disposición para el afrontamiento de situaciones } \\
\text { futuras }\end{array}$ \\
\hline
\end{tabular}

\section{RESULTADOS}

Los resultados se presentan organizados según los dos ejes temáticos principales. Primero, se describen los sentidos que los participantes atribuyeron a su diagnóstico de VIH, y después se muestran los cambios positivos que refieren haber experimentado en relación con dicho diagnóstico.

\section{Tema 1. Sentidos atribuidos al diagnóstico de VIH}

La mayoría consideró el diagnóstico como un punto de inflexión en sus vidas, afirmando que a partir de ese momento "hay un antes y un después... Hay un crac [sic] en la vida de una persona" (Participante 4 [P4]). Algunos interpretaron el diagnóstico como una nueva oportunidad o un renacer que les había brindado la posibilidad de llevar una vida diferente a la vivida hasta ese momento, de mejorar algunos aspectos de la misma o de iniciar una nueva etapa más positiva:
"Ya que uno tiene una segunda oportunidad de vivir [...] sería muy tonto de mi parte desaprovecharla" ( $\mathrm{P} 1)$.

"Esto es [...] como un volver a renacer. Como que murió una etapa de mi vida y vuelvo a renacer con otra" (P4).

Otros participantes expresaron haber podido capitalizar el diagnóstico como una situación de aprendizaje, concibiendo al VIH como la oportunidad de aprender algo nuevo y positivo acerca de ellos mismos y de su propia vida.

"El VIH ha sido un maestro en mi vida, porque me ha obligado a cuidarme a mí mismo" (P11).

"Fue como un obstáculo, una dificultad de la que yo hice un aprendizaje” (P10).

En otros casos, el VIH es sentido como una "situación límite" que más que una oportunidad o motivación para el cambio indica la necesidad de que este ocurra. En este caso, el diagnóstico permite al sujeto tomar conciencia de la posibilidad real y concreta de experimentar consecuencias 
negativas extremas. Los sujetos sienten que han "tocado fondo" y, en consecuencia, conciben al cambio como la única opción posible y como una necesidad perentoria de revertir el estilo de vida anterior al diagnóstico:

"La infección fue como una alarma: o te calmas, o cambias, o vas al muere. Y ahí algo me hizo clic [...] Sé que tal vez fue un freno para que no termine muerto" (P6).

"A veces algunos esperamos a caminar en el borde del precipicio para cambiar" (P8).

Algunos participantes se refieren al VIH como una consecuencia congruente con un estilo de vida llevado hasta el momento del diagnóstico. Aunque a primera vista los términos mencionados puedan ser interpretados como negativos (por ejemplo, castigo o descuido), parecen permitir al sujeto darle sentido al diagnóstico, facilitando su asimilación dentro de su trayectoria vital (por ejemplo, brindando una respuesta a la pregunta “¿Por qué a mí?”).

"Si cometiste el delito, tenés que cumplir la condena" (P14).

"Donde meti la pata, hay que afrontar" (P12).

Otros participantes reconocen al VIH como una condición crónica más que permite llevar una vida igual de funcional que la de cualquier otra persona. A esta percepción contribuye el conocimiento de la disponibilidad de un tratamiento eficaz. Asimismo, algunos participantes lo reevalúan contrastándolo con otras condiciones de salud, concluyendo que, en comparación, no es significativamente grave, incapacitante o letal, y que "Hay muchas enfermedades peores y muchas cosas peores" (P12). Ello facilita la desdramatización del diagnóstico y su naturalización:

"Una enfermedad terminal o un cáncer sí son un tema para que uno se tenga que preocupar, pero VIH... hay que pelearla” (P8).

"La vida de una persona con VIH es la vida de una persona normal que toma un remedio" (P12).

Finalmente, una proporción considerable de participantes reconoció que, en definitiva, adquirir el VIH es algo que le puede suceder a cualquier persona por el solo hecho de ser humano, independientemente de su estilo de vida o de conductas

Instituto de Investigaciones Psicológicas - Universidad Veracruzana ISSN impreso: 1405-1109 previas. Esto también facilita hallar una respuesta a la pregunta “¿Por qué a mí?”:

«Al principio te genera una cuestión de culpa y autocompasión, de decir “ ¿por qué a mi? ¿Qué hice yo?". Y después empezás a reconfigurar eso $y$ decís "Bueno, ¿por qué no?”» (P1).

\section{Tema 2. Cambios positivos atribuidos al diagnóstico}

Los cambios positivos englobados en este eje temático se agruparon en tres subtemas: Cambios en la filosofía de vida, Cambios vinculares y en la relación con los otros y Cambios en la percepción de sí mismos. Más de la mitad de los participantes refirió haber experimentado cambios positivos posteriores al diagnóstico de VIH, específicamente atribuidos a este evento. Muchos de estos cambios parecen estar relacionados con la atribución de sentidos más positivos al VIH.

\subsection{Cambios en la filosofía de vida:} reconfiguración de prioridades, objetivos y valores.

Los participantes refieren frecuentemente cambios profundos en la filosofía y el sentido de la propia vida. Lo que se refleja en un reordenamiento de sus prioridades y objetivos en la vida y en una reconfiguración de los valores que sustentaban sus decisiones:

"Senti que tenía que aprovechar el nuevo estado de las cosas para generar un cambio interno, en mi forma de vivir la vida" (P4).

2.1.1. Valor de la vida y el tiempo. Del relato de los participantes se infiere una revalorización de la vida y del tiempo por vivir ante el reconocimiento de que ambos, que antes se daban por sentados, pueden perderse. El diagnóstico puede, inicialmente, activar determinadas creencias vinculadas a la posibilidad de muerte y deterioro, por lo que puede incrementarse la conciencia acerca de la importancia del aprovechamiento del tiempo y del disfrute de la vida:

"Te das cuenta de que tenés una vida sola. No está para estar desperdiciando, me parece." (P1).

"Yo sentí que no tenía que perder el tiempo, porque a lo mejor no me quedaba mucho” (P11). 
2.1.2. Cambios en las actividades y en las prioridades. Los participantes señalaron cambios en sus prioridades y objetivos en la vida, así como en sus actividades. Afirman haber comenzado a preferir actividades placenteras y positivas que favorecen una reducción del estrés, generan bienestar emocional o implican crecimiento personal y autorrealización. Detrás de tal cambio se detecta la creencia de que la priorización de este tipo de actividades tendrá un resultado benéfico en la salud mental y emocional que, a largo plazo, también tendrá efectos positivos en la salud física y el bienestar general. En este sentido, es una estrategia para recuperar el control sobre el propio cuerpo y salud a partir de generar estados emocionales positivos y evitar el estrés. En contraste, aquellas actividades y obligaciones que les generaban malestar y estrés son ubicadas en segundo plano:

"Bajé un cambio [sic] y traté de alejarme de todo lo que me provocara estrés. Y conectarme un poco más con el lado positivo, con las cosas que a mí me hacen feliz para compensar el desequilibrio que me generaba la enfermedad" (P4).

En esta línea, algunos relatan haber retomado actividades postergadas. También mencionan que el diagnóstico los impulsó a reconectarse con las "pequeñas cosas" de la vida, es decir, situaciones sencillas y cotidianas. Ello implica el redescubrimiento y revalorización de esos aspectos significativos de sus vidas que antes recibían poca atención y que eran considerados como poco importantes o insignificantes, pues eran dados por sentados o naturalizados:

"Le empecé a dar más importancia a ciertas cosas [a las] que antes no le daba y que son las que en realidad me hacen un poco más feliz, lo que me produjo darle un montón de valor a cosas que antes eran insignificantes" (P14).

"Habia cosas que antes me habian hecho feliz a lo largo de mi vida, pero que había postergado... Conectarme con esas cosas que tenía relegadas" ( $\mathrm{P} 4)$.

"Cosas que por ahi tuviste antes y no las valorabas tanto [...] Yo creo que el VIH reconfigura todo eso. Decir: "Che, mirá todo lo que tenía” (P1).

De igual modo, los participantes refieren haber experimentado un reajuste en la valoración de la importancia y gravedad de las situaciones; esto

Instituto de Investigaciones Psicológicas - Universidad Veracruzana ISSN impreso: 1405-1109 es, un cambio en el sentido de redirigir sus preocupaciones, evitar hacerse problemas innecesariamente o atender cosas sin relevancia, y distinguir con mayor claridad un problema de una situación que realmente no lo es. Esto se condice con los esfuerzos activos por evitar situaciones estresantes y emocionalmente displacenteras que puedan afectar negativamente la salud mental y física:

"Trato de vivir cada día como si fuera el último... no te da mucho tiempo como para pensar "a ver en qué me voy a amargar"». (P11).

"Me hizo reconocer que la verdad no tengo problemas, porque el problema más grande, que fue ese, ya dejó casi de ser un problema; entonces, no hay problema más grave que ese” (P14).

2.1.3. Valor del cuerpo y la salud. De forma similar a lo señalado respecto a la vida y el tiempo, se aprecia una revalorización del propio cuerpo y de la salud, mismos que son reevaluados como prioritarios. Los temores y creencias vinculadas a la posibilidad de muerte y deterioro que puede activar el diagnóstico promueven también un aumento de la conciencia acerca de que el cuerpo y la salud no son recursos inagotables, que pueden perderse y que no deben darse por sentado. Algunos participantes refieren que el diagnóstico los llevó a reconsiderar los sentimientos de omnipotencia e invulnerabilidad que antes poseían:

"Empecé a quererme, a respetar mi cuerpo, porque sé que de este no hay otro. Si vos no te lo cuidas, no te lo va a cuidar nadie" (P8).

" "Avancé en darme cuenta de que tengo un solo cuerpo, y [que] es lo único que tengo en la vida, que lo tengo que cuidar. Perdí esa omnipotencia de que "A mi no me va a pasar". Soy consciente de que me puede pasar esta y cualquier otra cosa"》( (P2).

2.1.4. Incremento en el cuidado de la propia salud. Como resultado de lo anterior, se aprecia un incremento de las conductas de cuidado de la salud y el cuerpo y una priorización de actividades con ese fin. Muchos de los participantes informaron un aumento en la realización de conductas más saludables (por ejemplo, relacionadas con la alimentación y la actividad física) o el abandono de otras menos saludables (por ejemplo, ciertas prácticas sexuales o el uso de sustancias) a partir del diagnóstico: 
"Uno toma conciencia y decís "Bueno, voy a cuidar más mi cuerpo; voy a descansar mejor, voy a alimentarme mejor, no lo voy a maltratar tanto", como lo hacía antes» (P1).

"Yo qué gano saliendo a emborracharme, a estar de vuelta con un tipo o con otro. Si no me cuido, puedo estar igual o estar peor; entonces trato de llevar una vida sana” (P8).

\subsection{Cambios vinculares y en la relación con los otros}

Los participantes refieren cambios positivos respecto a los vínculos y relaciones con otras personas. En general, reportaron mayor intercambio y expresión emocional; más cercanía, conexión y profundidad; el fortalecimiento de algunos vínculos y la priorización de aquellos que eran más positivos y enriquecedores.

2.2.1. Cambios en la priorización de los vínculos y mayor expresión e intercambio emocional. Así como los participantes indican haber revalorizado las "pequeñas cosas de la vida" mencionan haber hecho lo mismo con la importancia que tienen los demás en su vida, que antes era naturalizada. Esto favorece una mayor conciencia de la relevancia de demostrar a los otros cuán importantes y significativos son, y de compartir abiertamente con ellos sus emociones, algo que antes del diagnóstico se encontraba naturalizado. El resultado es un aumento de la expresión y el intercambio emocional positivo, que conlleva una mayor empatía y capacidad para reconocer las propias emociones:

"A mí me cambió mucho la cabeza con respecto a mí: reconocerme como una persona con emociones, con sentimientos, y al otro también registrarlo como una persona que tiene sentimientos, que tiene emociones" (P1).

Por otro lado, así como se reestructuran los objetivos y las prioridades en la vida, también se reconfiguran las prioridades vinculares. Se aprecia una mayor selectividad en las relaciones interpersonales y la preferencia por vínculos más positivos que implican un intercambio de emociones positivas y placenteras y un mutuo enriquecimiento. En contraste, se descartan otros que se perciben como negativos o que producían malestar emocional:
"Tuve que hacer una limpieza y una selección... Amistades hoy en día... cinco es mucho. En las jodas estamos todos, y cuando te pasa una desgracia se borran todos... Con cinco es suficiente" (P8).

"También hacer una gran selección de la gente con la que uno se rodea. Cuando alguien no me cierra, ¿por qué tengo que estar al lado de esta persona? Entonces prefiero evitarla” (P14).

2.2.2. Fortalecimiento de vínculos cercanos y significativos. Según los participantes, lo anterior contribuyó a una mayor conexión, intimidad y cercanía emocional con los otros y a establecer vínculos más profundos. También reportan un aumento de la confianza mutua, atribuido en parte al hecho de compartir con la otra persona información sensible, como el diagnóstico. En suma, estos cambios han conducido a fortalecer algunos vínculos significativos en sus vidas:

"Creo que esto ayudó a la relación con los demás. Ayudó un poco al vínculo familiar” (P2).

"Un cambio de mayor apertura, positivo. De darme las gracias por compartir, por confiar. Se abre todo un vínculo, se hace más profundo; se siente como que le estás dando algo que es íntimo. La gente siente confianza y lo valora (P10).

2.2.3. Fortalecimiento de la red de apoyo: aceptación de la ayuda de otros. La percepción de que el diagnóstico puede generar la pérdida de la salud promueve una mayor conciencia de las propias limitaciones y la comprensión de que la ayuda de los demás en ocasiones resulta necesaria. Una mayor receptividad y disposición para aceptar la ayuda de las personas puede contribuir a fortalecer la red de apoyo social del sujeto:

"Uno toma conciencia de eso. No es decir "Yo soy solo y estoy yo solo o puedo solo". Hay momentos que uno también necesita apoyarse en el otro o necesita la compañía del otro; en ese sentido, creo que mis vínculos cambiaron» (P1).

Esto se relaciona en parte con que, en respuesta a compartir el diagnóstico, las personas significativas para los participantes han demostrado un mayor apoyo y preocupación respecto de la salud y bienestar de estos, dando como resultado un aumento en el esfuerzo por protegerlos, asistirlos o cuidarlos: 
"Fue doloroso contarlo en mi familia, pero a veces la salud es un tema más por el que los demás se preocupan por uno" (P2).

"[Noté en ellos] un cambio en el sentido de querer protegerme" (P11).

\subsection{Cambios en la percepción de sí mismos}

2.3.1. Nueva conciencia de las propias fortalezas $y$ recursos para el afrontamiento. Los participantes relatan que haber enfrentado la situación de diagnóstico les hizo posible descubrir capacidades en ellos mismos que desconocían, modificando así la percepción que tenían de sí mismos. Entre ellas, describen el descubrimiento de una fortaleza interna, que ellos mismos no eran conscientes de que poseían, para afrontar exitosamente las dificultades. El resultado fue un autoconocimiento más profundo en cuanto a reconocerse más fuertes, resilientes y con una mayor percepción de autoeficacia o autoconfianza para afrontar adaptativamente las situaciones problemáticas:

"Uno se empieza a sentir fuerte. Y la fortaleza está en uno. Uno se da cuenta que está todo bien" (P10).

"Pienso que voy a superar todos estos traspiés, que voy a salir fortalecido. Esta situación, como aspecto positivo, me ha hecho crecer" (P4).

2.3.2. Disposición para afrontar situaciones futuras. Asimismo, confían en poder generalizar este fortalecimiento al comprender que este aprendizaje o nuevo recurso a su disposición les permitirá afrontar mejor situaciones futuras. Lo anterior, en combinación con los otros cambios positivos ya mencionados, permite acrecentar la sensación de control sobre el propio cuerpo, la salud y la propia vida:

"Empecé a tomar las riendas de mi vida"(P10).

\section{DISCUSIÓN}

El objetivo del presente estudio fue explorar los sentidos atribuidos al VIH y la experimentación de cambios positivos posteriores al diagnóstico en hombres gays residentes en el Área Metropolitana de Buenos Aires (AMBA), Argentina. Los resultados muestran que la mayoría de los participan-

Instituto de Investigaciones Psicológicas - Universidad Veracruzana ISSN impreso: 1405-1109 tes reportó haber experimentado cambios positivos que atribuían al diagnóstico de $\mathrm{VIH}$, encuadrables dentro del concepto de crecimiento postraumático, lo que parece estar relacionado con atribuir al VIH sentidos más positivos y que favorecen el cambio, tal como proponen las teorías del CPT en cuanto al ajuste al diagnóstico de condiciones de salud crónicas (Park, 2013; Sawyer et al., 2010). Los cambios identificados se organizaron en tres temas o ejes: Cambios en la filosofía de vida (reconfiguración de prioridades, objetivos y valores), Cambios vinculares y en la relación con los otros y Cambios en la percepción de sí mismos (conciencia de las propias fortalezas y recursos para el afrontamiento). Estos resultados son altamente consistentes con los hallazgos de estudios internacionales acerca de CPT en hombres gays con VIH en los que se empleó una metodología similar (Siegel y Schrimshaw, 2000a).

Un conjunto de cambios observados incluye modificaciones en la filosofía de vida, que implican una reorganización de las prioridades, metas y valores. Se aprecian dos consecuencias principales: la priorización de actividades placenteras y una mayor realización de conductas de cuidado de la propia salud o el abandono de las poco saludables. Siegel y Schrimshaw (2000a; 2000b) explican estos cambios como una estrategia para recuperar la sensación de control sobre la propia salud, que se percibe perdida como resultado del diagnóstico. El involucramiento en actividades placenteras y eludir actividades estresantes se basan en creencias acerca de la relación mente-cuerpo, y en que promover una actitud mental positiva puede tener un impacto benéfico en la propia salud al potenciar, por ejemplo, la respuesta inmune. A ello contribuye también eliminar o evitar conductas como fumar, consumir alcohol u otras sustancias, así como tener relaciones sexuales no seguras, lo que promueve la sensación de estar fortaleciendo activamente la propia salud. Por ejemplo, Wang et al. (2017) mostraron que el CPT se asoció con menos sexo anal sin protección con parejas ocasionales en HSH recientemente diagnosticados con VIH. La sensación de control sobre los eventos vitales ha sido identificada como uno de los componentes del bienestar subjetivo (Ryff, 1989) y como un recurso para afrontar adaptativamente el estigma 
relacionado con el VIH y con la orientación sexual en hombres gays de Argentina (Arístegui et al., 2017).

Otro grupo de cambios se observó en relación con los vínculos y las relaciones interpersonales. En consistencia con los hallazgos de Siegel y Schrimshaw (2000a), los participantes reportaron un mayor aprecio por la relación con otras personas significativas, dando prioridad a los vínculos valorados como más positivos o enriquecedores. Esto redundó en una mayor cercanía, intercambio emocional y disposición a aceptar la ayuda de los demás. Estudios previos han mostrado que poder dar un sentido al diagnóstico, incrementando la percepción del valor y el propósito de la propia vida, puede afectar favorablemente la percepción y disponibilidad de apoyo social (Farber, Lamis, Shahane y Campos, 2014). Este último ha sido identificado como un factor protector que modera el efecto negativo del estigma sobre la salud mental en los HSH (Yan et al., 2019). En esta misma línea, estudios locales han mostrado que la aceptación de la orientación sexual y del diagnóstico de VIH por parte de los otros, así como los vínculos interpersonales de calidad, promueven el bienestar subjetivo y son recursos para afrontar el estigma y la discriminación en hombres gays con VIH (Arístegui et al., 2017).

Un tercer conjunto de cambios se refiere a la redefinición de la percepción de sí mismos, en el sentido de adquirir una mayor conciencia de las propias fortalezas y capacidades para afrontar las adversidades. El aumento del sentido de fortaleza personal se ha reconocido como un componente del CPT desde la propuesta inicial de Tedeschi y Calhoun (2004). En línea con lo apuntado previamente, también puede estar relacionado con la restauración de la sensación de control sobre los eventos de la propia vida, y es asimismo un recurso valioso para el afrontamiento adaptativo de eventos adversos, como los episodios de estigma y discriminación (Arístegui et al., 2017).

Estos resultados refuerzan la evidencia de que el otorgamiento de sentidos más positivos o constructivos al VIH favorece la aceptación del diagnóstico y el ajuste psicológico posterior al promover cambios positivos en los sujetos (Fife, 2005; Sawyer et al., 2010). Como han subrayado Siegel

Instituto de Investigaciones Psicológicas - Universidad Veracruzana ISSN impreso: 1405-1109 y Schrimshaw (2000a) y Farber et al. (2014), encontrarle un sentido o atribuirle un propósito al evento dentro del curso de la propia vida puede tornarlo más aceptable y promover el CPT. Incluso algunas creencias vinculadas a la responsabilidad y el merecimiento, contrariamente a lo que se esperaría, pueden favorecer el hallazgo de un sentido al diagnóstico de VIH y promover su aceptación, tal como sugieren los resultados de este estudio y de otros (Plattner y Meiring, 2006).

No obstante, es importante destacar que el CPT no implica la ausencia de malestar emocional. De hecho, el CPT es desencadenado por los esfuerzos para afrontar el malestar disparado por el evento traumático o estresante. Es por ello que los individuos pueden atravesar cambios positivos aunque continúen experimentando niveles significativos de emocionalidad negativa en forma simultánea (Tedeschi y Calhoun, 2004; Yu et al., 2017). Si bien los participantes de este estudio refirieron cambios encuadrables dentro del concepto de CPT, también expresaron haber experimentado un malestar emocional intenso como resultado del diagnóstico, lo que ya fue expuesto con anterioridad (Radusky y Mikulic, 2018).

Es necesario reconocer las limitaciones de este estudio. En primer lugar, el método de muestreo por conveniencia puede haber generado sesgos en la selección de los participantes. Por ejemplo, es posible apreciar que los sujetos incluidos en la muestra tenían, en general, un nivel educativo elevado, por lo que no fueron necesariamente representativos de la totalidad de los hombres gays residentes en la citada localidad. El sesgo por deseabilidad social también puede haber influido en las respuestas de los participantes al intentar destacar estrategias de afrontamiento ante el diagnóstico más adaptativas, mostrando una imagen de sí mismos más positiva ante el entrevistador. De igual manera, hay limitaciones inherentes a la metodología cualitativa elegida. Futuros estudios podrán beneficiarse de llevar a cabo un muestreo que controle en mayor medida la ocurrencia de sesgos, y del uso de múltiples metodologías que combinen los enfoques cualitativo y cuantitativo.

A pesar de lo anterior, estos resultados apor$\tan$ nueva evidencia sobre los mecanismos que favorecen el CPT en hombres gays con VIH, y con- 
tribuyen a llenar el vacío investigativo sobre este tópico en el contexto latinoamericano. La información obtenida muestra que resulta recomendable incorporar componentes que potencien el CPT en las intervenciones psicosociales y psicoterapéuticas dirigidas a la referida población. Los resultados de este trabajo pueden ser útiles para el diseño de intervenciones más focalizadas y ajustadas al identificar las áreas vitales en las que el CPT habitualmente puede ocurrir. La relevancia de este tipo de abordajes más integrales radica en que los cambios positivos que pueden encuadrarse en el concepto de CPT pueden ser recursos para afrontar efectivamente el estigma y la discriminación por diversos motivos. Asimismo, tales cambios promueven una mayor salud mental al facilitar la aceptación del diagnóstico y el ajuste psicológico posterior. Por este motivo, también pueden favorecer la adherencia al tratamiento antirretroviral, la retención en la atención del VIH y el logro de una carga viral indetectable, lo que mejora la salud física y la calidad de vida de quienes viven con el VIH y reduce la posibilidad de su transmisión a otras personas.

Citación: Radusky, P.D., Zalazar, V. y Arístegui, I. (2022). Crecimiento postraumático en hombres gays con VIH en Buenos Aires, Argentina. Psicología y Salud, 32(1), 93-104. https:// Doi.org/10.25009/pys.v32i1.2716.

\section{REFERENCIAS}

Adams, C., Zacharia, S., Masters, L., Coffey, C. y Catalan, P. (2016). Mental health problems in people living with HIV: Changes in the last two decades: The London experience 1990-2014. AIDS Care, 28, 56-59. Doi: 10.1080/09540121.2016.1146211.

Arístegui, I., Radusky, P.D., Zalazar, V., Lucas, M. y Sued, O. (2017). Resources to cope with stigma related to HIV status, gender identity, and sexual orientation in gays men and transgender women. Journal of Health Psychology, 23(2), 320-331. Doi: $10.1177 / 1359105317736782$.

ATLAS.Ti(v. 7.5.4) [software](2015). Recuperado de https://atlasti.cleverbridge.com/74/purl-order?_ga=2.43624933.1185314242. $1523370337-1733085140.1523370337$.

Bassett, S.M., Schuette, S.A., O’Dwyer, L.C. y Moskowitz, J.T. (2019). Positive affect and medication adherence in chronic conditions: A systematic review. Health Psychology, 38(11), 960-974. Doi: 10.1037/hea0000778.

Bonanno, G.A. (2004). Loss, trauma, and human resilience: Have we underestimated the human capacity to thrive after extremely aversive events? American Psychologist, 59(1), 20-28. Doi: 10.1037/0003-066x.59.1.20.

Braun, V. y Clarke, V. (2006). Using thematic analysis in psychology. Qualitative Research in Psychology, 3(2), 77-101. Doi: 10.1191/1478088706qp063oa.

Camargo C., C., Cavassan N., R.V., Tasca K., I., Meneguin, S., Miot, H.A. y Souza, L.R. (2019). Depression and coping are associated with failure of adherence to antiretroviral therapy among people living with HIV/AIDS. AIDS Research and Human Retroviruses, 35(11-12), 1181-1188. Doi: 10.1089/aid.2019.0050.

Farber, E.W., Lamis, D.A., Shahane, A.A. y Campos, P.E. (2014). Personal meaning, social support, and perceived stigma in individuals receiving HIV mental health services. Journal of Clinical Psychology in Medical Settings, 21(2), 173-182. Doi: 10.1007/s10880-014-9394-3.

Federación Argentina LGBT (2019). Observatorio de crímenes de odio LGBT. Informe 2019. Recuperado de https://falgbt.org/ crimenes-de-odio/.

Fife, B.L. (2005). The role of constructed meaning in adaptation to the onset of life-threatening illness. Social Science and Medicine, 61(10), 2132-2143. Doi: 10.1016/j.socscimed.2005.04.026.

Garrido H., H., Murphy, P.J. y Alonso T., J. (2017). Predictors of resilience and posttraumatic growth among people living with HIV: A longitudinal study. AIDS and Behavior, 21(11), 3260-3270. Doi: 10.1007/s10461-017-1870-y.

Jeffries, W.L., Townsend, E.S., Gelaude, D.J., Torrone, E.A., Gasiorowicz, M. y Bertolli, J. (2015). HIV stigma experienced by young men who have sex with men (MSM) living with HIV infection. AIDS Education and Prevention, 27(1), 58-71. Doi: 10.1521/aeap.2015.27.1.58.

Krasnow, A.N. (2012). El nuevo modelo de matrimonio civil en el derecho argentino. Revista de Derecho Privado, 22.

Ley de Matrimonio Civil (Matrimonio Igualitario) (2010). Boletín Oficial de la Nación N²6.618. Buenos Aires.

Martin, L. y Kagee, A. (2011). Lifetime and HIV-related PTSD among persons recently diagnosed with HIV. AIDS and Behavior, 15(1), 125-131. Doi: 10.1007/s10461-008-9498-6.

Milam, J.E. (2004). Posttraumatic growth among HIV/AIDS patients. Journal of Applied Social Psychology, 31(11), 2353-2376.

Instituto de Investigaciones Psicológicas - Universidad Veracruzana

ISSN impreso: 1405-1109
Psicología y Salud, Vol. 32, Núm. 1: 93-104, enero-junio de 2022

https://doi.org/10.25009/pys.v32i1.2716 
Milam, J.E. (2006). Posttraumatic growth and HIV disease progression. Journal of Consulting and Clinical Psychology, 74(5), 817-827. Doi: 10.1037/0022-006x.74.5.817.

Ministerio de Salud de la Nación (2019). Boletín sobre el VIH, sida e ITS en la Argentina, Nº36. Recuperado de http://www.msal. gob.ar/images/stories/bes/graficos/0000001754cnt-boletin-epidemiologico-2019_vih-sida-its.pdf.

Murphy, P.J. y Hevey, D. (2013). The relationship between internalised HIV-related stigma and posttraumatic growth. AIDS and Behavior, 17(5), 1809-1818. Doi: 10.1007/s10461-013-0482-4.

Nightingale, V.R., Sher, T.G. y Hansen, N.B. (2010). The impact of receiving an HIV diagnosis and cognitive processing on psychological distress and posttraumatic growth. Journal of Traumatic Stress, 23(4), 452-460. Doi: 10.1002/jts.20554.

Park, C.L. (2013). The Meaning Making Model: A framework for understanding meaning, spirituality, and stress-related growth in health psychology. Bulletin of the European Health Psychology Society, 15(2), 40-47. Recuperado de https://www.ehps. net/ehp/index.php/contents/article/view/ehp.v15.i2.p40/1041.

Plattner, I.E. y Meiring, N. (2006). Living with HIV: The psychological relevance of meaning making. AIDS Care, 18(3), 241-245. Doi: $10.1080 / 09540120500456227$.

Radusky, P.D. y Mikulic, I.M. (2018). Impacto emocional del diagnóstico de VIH en personas residentes en Buenos Aires. Anuario de Investigaciones, 25, 107-116.

Radusky, P.D. y Mikulic, I.M. (2019). Experiencia y percepción de estigma relacionado con el VIH en varones con orientación homosexual. Salud y Sociedad, 10(2), 110-127. Doi: 10.22199/issn.0718-7475-2019-02-010.

Ryff, C.D. (1989). Happiness is everything, or is it? Explorations on the meaning of psychological well-being. Journal of Personality and Social Psychology, 57(6), 1069-1081. Doi: 10.1037/0022-3514.57.6.1069.

Rzeszutek, M., Oniszczenko, W. y Firląg-Burkacka, E. (2016). Gender differences in posttraumatic stress symptoms and the level of posttraumatic growth among a Polish sample of HIV-positive individuals. AIDS Care, 28(11), 1411-1415. Doi: 10.1080/09540121.2016.1182615.

Sawyer, A., Ayers, S. y Field, A.P. (2010). Posttraumatic growth and adjustment among individuals with cancer or HIV/AIDS: A meta-analysis. Clinical Psychology Review, 30(4), 436-447. Doi: 10.1016/j.cpr.2010.02.004.

Siegel, K. y Schrimshaw, E.W. (2000a). Coping with negative emotions: The cognitive strategies of HIV-infected gays/bisexual men. Journal of Health Psychology, 5(4), 517-530. Doi: 10.1177/135910530000500409.

Siegel, K. y Schrimshaw, E.W. (2000b). Perceiving benefits in adversity: Stress-related growth in women living with HIV/AIDS. Social Science and Medicine, 51(10), 1543-1554. Doi: 10.1016/s0277-9536(00)00144-1.

Tedeschi, R.G. y Calhoun, L.G. (2004). Posttraumatic growth: Conceptual foundations and empirical evidence. Psychological Inquiry, 15(1), 1-18. Doi: 10.1207/s15327965pli1501.

Theuninck, A.C., Lake, N. y Gibson, S. (2010). HIV-related posttraumatic stress disorder: Investigating the traumatic events. AIDS Patient Care and STDS, 24(8), 485-491. Doi: 10.1089/apc.2009.0231.

Wang, Z., Wu, X., Lau, J.T.F., Mo, P.K.H., Mak, W.W.S., Wang, X., Yang, X., Gross, D. y Jiang, H. (2017). Prevalence of and factors associated with unprotected anal intercourse with regular and nonregular male sexual partners among newly diagnosed HIV-positive men who have sex with men in China. HIV Medicine, 18(9), 635-646. Doi: 10.1111/hiv.12500.

Willie, T.C., Overstreet, N.M., Peasant, C., Kershaw, T., Sikkema, K.J. y Hansen, N.B. (2016). Anxiety and depressive symptoms among people living with HIV and childhood sexual abuse: The role of shame and posttraumatic growth. AIDS and Behavior, 20(8), 1609-1620. Doi: 10.1007/s10461-016-1298-9.

Yan, H., Li, X., Li, J., Wang, W., Yang, Y., Yao, X., Yang, N. y Li, S. (2019). Association between perceived HIV stigma, social support, resilience, self-esteem, and depressive symptoms among HIV-positive men who have sex with men (MSM) in Nanjing, China. AIDS Care, 31(9), 1069-1076. Doi: 10.1080/09540121.2019.1601677.

Yu, N.X., Chen, L., Ye, Z., Li, X. y Lin, D. (2017). Impacts of making sense of adversity on depression, posttraumatic stress disorder, and posttraumatic growth among a sample of mainly newly diagnosed HIV-positive Chinese young homosexual men: the mediating role of resilience. AIDS Care, 29(1), 79-85. Doi: 10.1080/09540121.2016.1210073. 